\title{
ESTUDIO SECTORIAL PARA UN PROYECTO DE PARAJE NATURAL MUNICIPAL EN EL CASTILLO DE SAX (ALICANTE)
}

\author{
Fernando Tomás Maestre Gil \\ Vicente José Pascual Abellán \\ Departamento de Ecología. Universidad de Alicante
}

\section{RESUMEN}

Se analiza en este trabajo una propuesta para la declaración de un paraje natural municipal en el Castillo de Sax (Alicante), figura legal recogida en la ley autonómica 11/1994, de 27 de diciembre, de Espacios Naturales Protegidos de la Comunidad Valenciana. La importancia local del paraje y la necesidad de su protección queda puesta de manifiesto tras el análisis de su medio físico, ya que alberga en una superficie inferior a 10 ha 226 táxones vegetales (de los que 25 son endémicos), 23 sintáxones vegetales, 9 hábitats de interés comunitario y 21 especies de vertebrados, así como numerosos recursos culturales, entre los que destacan el Castillo de Sax y diversos yacimientos arqueológicos pertenecientes a la Edad del Bronce y a épocas romana y medieval. La presencia de vertederos de residuos sólidos, la degradación del hábitat de diversas comunidades vegetales y la presencia de procesos erosivos han sido identificados como los principales problemas ambientales. Frente a otras figuras recogidas en la ley 11/1994, la de paraje natural municipal, que permite a las corporaciones municipales proponer para su declaración y gestionar aquellos espacios naturales de interés local presentes en su territorio, se muestra como la más apropiada para la conservación de este espacio natural.

Palabras clave: conservación, espacio natural protegido, paraje natural municipal, Castillo de Sax, Alicante.

\begin{abstract}
In this work we explore the possibilities that Castillo de Sax natural area (NW Alicante) has to receive legal protection within the «paraje natural municipal» category, a protection figure present within the Valencia Region Protected Areas Law (law 11/1994). The analysis of the environment in the study area reflects the presence of 226 vegetal taxa ( 25 of them are exclusive of SE Spain), 23 plant communities, 9 habitats included in the European Directive 92/43/CEE, and 21 vertebrate species, together with a rich cultural patrimony based mainly on the presence of an Arab Castle and several Bronze Age, Roman Age and Middle Ages archaeological sites. The presence of rubbish dumps, habitat degradation and erosion have
\end{abstract}


been identified as the main environmental problems. Between the different classes of protected areas that the law 11/1994 reflect, the category of «paraje natural municipal» allows to local town councils to manage natural areas of interest that lie within their townships, and is the most suitable for the protection of the study area.

Key words: Natural protected area, Conservation, Castillo de Sax, Alicante.

\section{Introducción}

Dentro de las distintas estrategias para la conservación de la biodiversidad, el establecimiento de Espacios Naturales Protegidos (en adelante ENP) es una de las medidas que mayor importancia está cobrando en la actualidad. Dentro del panorama español, la transferencia de las competencias en materia de conservación de la naturaleza a las comunidades autónomas, el creciente interés por las cuestiones ambientales y la creación de un marco jurídico apropiado han incrementado considerablemente el establecimiento de ENP durante las dos últimas décadas como herramienta básica de conservación y gestión de aquellos espacios naturales de mayor interés (Llorens y Rodríguez, 1991; Mulero, 1999).

Mediante el R.D. 2365/1984 se produjo el traspaso de las competencias en materia de conservación de la naturaleza a la Comunidad Valenciana. Fruto de este traspaso es la aprobación por parte de esta comunidad autónoma de la ley 5/1988, de 24 de junio, reguladora de los Parajes Naturales en la Comunidad Valenciana. Esta normativa contemplaba la creación de una nueva figura de protección, el paraje natural, complementaria a las cuatro categorías de ENP recogidas en la entonces vigente ley 11/75, de Espacios Naturales Protegidos (reserva integral de interés científico, parque nacional, paraje natural de interés nacional y parque natural). La promulgación de la ley 4/89, de 27 de marzo, de Conservación de los Espacios Naturales y de la Flora y Fauna Silvestre - nacida al amparo de las competencias que el Estado tiene en la redacción de una legislación básica en materia de medio ambiente-, supuso la introducción de nuevas figuras de protección (parque, reserva natural, paisaje protegido y monumento natural), que intentaban evitar la disparidad de criterios que se empezaba a poner de manifiesto en la legislación autonómica (Mulero, 1999).

El uso ordinario de la Ley de Parajes Naturales de la Comunidad Valenciana puso de manifiesto diversas necesidades jurídicas y administrativas que esta norma no podía capaz de resolver satisfactoriamente (Generalitat Valenciana, 1996). Este hecho, unido a la necesidad de adecuar a la realidad territorial de esta comunidad la ley 4/89 y las directivas europeas 91/294/CEE (referente a la Directiva de Aves Silvestres) y 92/43/CEE (Directiva de Hábitats), propició la creación de la Ley de Espacios Naturales Protegidos de la Comunidad Valenciana (ley 11/1994, de 27 de diciembre). Entre las categorías de ENP introducidas por esta norma destaca la de paraje natural municipal, figura que permite a los propios ayuntamientos proponer para su declaración y gestionar aquellos espacios naturales de interés local presentes en su territorio. Esta capacidad que se otorga a los municipios es una de las novedades más interesantes que introduce esta norma, ya que permite agilizar considerablemente los trámites para la declaración del ENP, a la vez que se concede a las corporaciones locales un papel relevante en la conservación del patrimonio natural acorde con la importancia que este ámbito de actuación tiene dentro de las actividades de conservación y gestión ambiental (Keen y Mercer, 1993; Kenny-Gilday et al., 1995; Garrod y Willis, 1994). 
En el presente artículo se aborda una propuesta para la creación de un paraje natural municipal en el Castillo de Sax (Alicante) y su entorno. La importancia ecológica de este paraje ya ha sido puesta de manifiesto en trabajos anteriores (Maestre, 1998a, 1998b, 1999a, 2000a, 2000b), aunque todavía no se ha abordado el análisis detallado de su medio físico ni su posible declaración como espacio protegido. El principal objetivo de este estudio es servir de base para una futura propuesta oficial de paraje natural municipal. Para ello se aborda el análisis de su medio físico y de los principales impactos ambientales que presenta, discutiendo la idoneidad de esta categoría de protección y otras figuras alternativas que también podrían emplearse.

\section{Materiales y métodos}

\section{a) Zona de estudio}

El Castillo de $\operatorname{Sax}\left(38^{\circ} 32^{\prime}\right.$ de latitud y $0^{\circ} 49^{\prime} \mathrm{W}$ de longitud) se encuentra situado en el extremo noroccidental de la provincia de Alicante, a unos $44 \mathrm{~km}$ de la capital de la provincia (figura 1). Con un área de 9.82 ha, la zona de estudio comprende el macizo rocoso conocido con el nombre de «La Peña» (558 m de altitud) y la ladera que lo acompaña en su cara norte (foto 1 ).

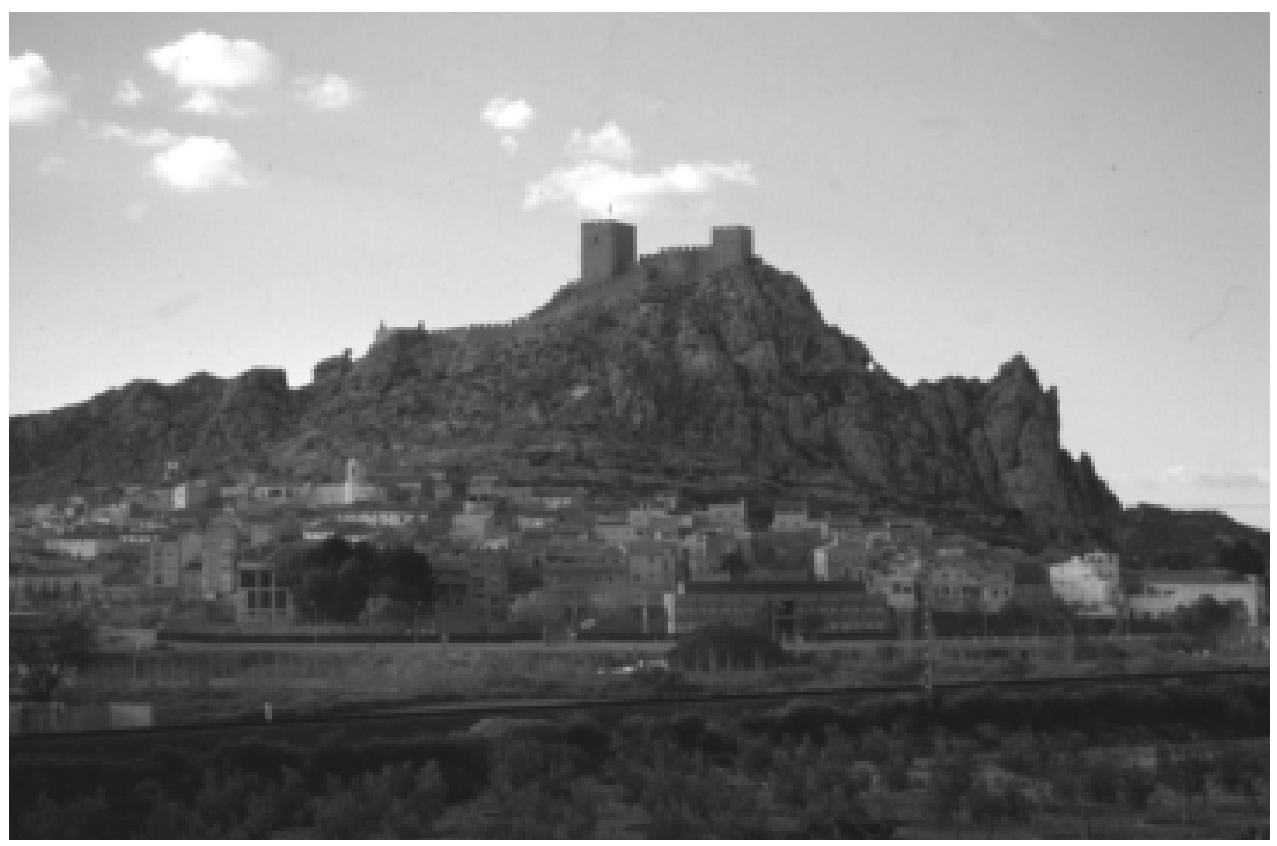

Fото 1. Vista parcial de la parte sur del roquedo y del casco urbano. 

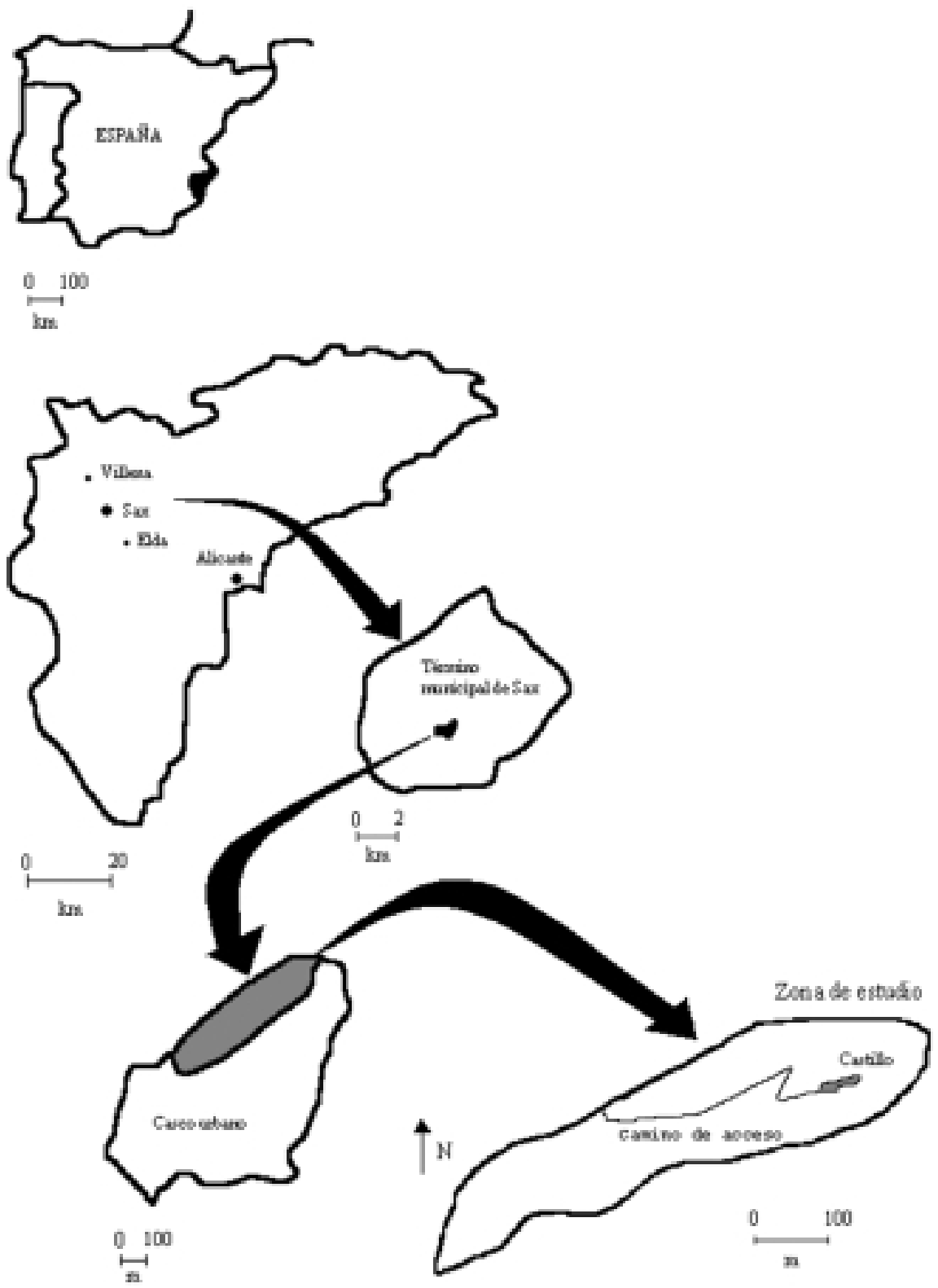

FIGURA 1. Localización y dimensiones de la zona de estudio. 
A la hora de elegir los distintos elementos del medio físico a inventariar, se ha seguido la clasificación propuesta por la Secretaría General de Medio Ambiente (1996), eligiéndose entre todas las variables recogidas en esta obra las siguientes: climatología, geología, edafología, flora y vegetación, fauna, recursos culturales y riesgos geológicos. También se recoge dentro de este epígrafe el estudio de los hábitats de interés comunitario recogidos en la Directiva 92/42/CEE, traspuesta al ordenamiento jurídico español mediante el R.D. $1997 / 1995$.

Los datos climáticos utilizados provienen de Maestre (2000a). Para el establecimiento de la geología se ha empleado el Mapa Geológico a escala 1:50.000 (IGME, 1978) y las informaciones recogidas en Ponce (1987). La tipificación del suelo se ha elaborado siguiendo la nomenclatura propuesta por la FAO-UNESCO (1988) recogida en De la Torre y Alías (1996), complementando la información aquí mostrada con observaciones de campo.

Los muestreos relacionados con el estudio de la flora, vegetación y hábitats se han realizado durante el período 1994-1998. El estudio florístico se basa en material herborizado durante este tiempo, correspondiendo la nomenclatura y autoría de los táxones vegetales con los que aparecen en Mateo y Crespo (1998). Para describir la vegetación se ha empleado la metodología fitosociológica de la escuela Zürich-Montpellier (Braun-Blanquet, 1979), recurriendo a la comparación de inventarios fitosociológicos levantados sobre el terreno durante 1998 con tablas de autores precedentes (Alcaraz et al., 1991; Alonso, 1996; De la Torre et al., 1997). En el caso de los hábitats de interés comunitario, se ha considerado como tal a toda comunidad vegetal incluida en el Anexo I del R. D. 1997/1995.

En el estudio faunístico se incluyen todas aquellas especies de vertebrados encontradas en la zona de estudio durante el período 1994-1998, detectadas por observación directa o por la presencia de restos, excrementos o huellas.

Los recursos culturales catalogados en este trabajo son los arqueológicos, los históricos, los etnográficos y los científico-educativos, de acuerdo con la clasificación propuesta por la Secretaría General de Medio Ambiente (1996). Para ello se ha recurrido fundamentalmente a la consulta de diversas fuentes bibliográficas.

Dentro de los riesgos geológicos se analizan la erosión y los desprendimientos. El estudio de los procesos erosivos se ha llevado a cabo sobre el terreno, considerando únicamente las manifestaciones provocadas por la erosión hídrica en superficie, sin distinción entre las distintas formas que ésta puede presentar. En el caso de los desprendimientos, se aborda la problemática de la caída de rocas que han tenido lugar en la zona de estudio durante la última década y las actuaciones ejecutadas para evitar futuros desplomes.

\section{c) Impactos ambientales}

Los principales impactos ambientales que afectan al paraje estudiado son los vertederos ilegales de residuos sólidos, la presencia de procesos erosivos y la destrucción del hábitat natural de diversas comunidades vegetales por las obras de infraestructura efectuadas en su entorno.

Los datos utilizados se basan en muestreos esporádicos realizados entre febrero de 1998 y enero de 2000, donde se recogió información sobre los vertederos ilegales (localización, extensión y residuos depositados en ellos), las zonas donde aparecen fenómenos erosivos y aquellos lugares donde se ha alterado el hábitat natural. 


\section{Descripción del medio físico}

a) Geología, edafología y climatología

La litología de la zona de estudio se caracteriza por la dominancia de calizas y margas (figura 2A), estando también presente las arcillas y yesos rojos del Keuper, que forman parte de un gran afloramiento de carácter diapírico que llega hasta la provincia de Albacete. El macizo rocoso que domina la zona de estudio se originó en los plegamientos que tuvieron lugar durante el Mioceno Medio, hace 20 millones de años (figura 2B). Los materiales más antiguos que lo constituyen son las arcillas grises que aparecen en la base, que cuentan con una edad de 58 millones de años; después se encuentran las calizas, que constituyen la parte culminante de «La Peña». Materiales más recientes son las calizas margosas, con unos 25 millones de años, que en la actualidad no aparecen en superficie y que se encuentran debajo del emplazamiento del casco urbano. Por último aparece un estrato de calizas arenosas de unos 20 millones de años de antigüedad, oculto también bajo el caserío. Durante todo el Cuaternario, las rocas de «La Peña» se han visto sometidas a procesos erosivos que han ido depositando estos materiales en las faldas del roquedo (Ponce, 1987).

Desde el punto de vista climático, los datos para la localidad de Sax arrojan una temperatura media anual de $16.4{ }^{\circ} \mathrm{C}$ y unas precipitaciones medias anuales de $315 \mathrm{~mm}$, lo que le otorgan un ombroclima semiárido (tabla 1). El balance hídrico refleja una elevada demanda evaporativa, superior a las precipitaciones durante la mayor parte del año, dando lugar a la aparición de un déficit hídrico especialmente acusado durante la época estival. Si bien estas características son para la localidad en su conjunto, dentro de la zona estudiada existen condiciones microclimáticas particulares provocadas por la existencia de «La Peña». Este macizo rocoso va a modificar los valores de insolación y humedad existentes según la orientación que se considere, existiendo notables diferencias entre la zona de

Tabla 1

RESUMEN DE LOS PRINCIPALES PARÁMETROS CLIMÁTICOS Y BALANCE HÍDRICO PARA LA LOCALIDAD DE SAX (OBSERVATORIO SITUADO A O.5 KM DE LA ZONA DE ESTUDIO) EN EL PERÍODO 1986-1995

\begin{tabular}{|c|c|c|c|c|c|c|c|c|c|c|c|c|}
\hline & $\mathbf{E}$ & $\mathbf{F}$ & $\mathbf{M}$ & $\mathbf{A}$ & $\mathbf{M y}$ & $\mathbf{J}$ & $\mathbf{J l}$ & $\mathbf{A}$ & $\mathbf{S}$ & $\mathbf{0}$ & $\mathbf{N}$ & $\mathbf{D}$ \\
\hline $\mathrm{P}$ & 12.58 & 26.80 & 26.01 & 31.69 & 29.51 & 41.92 & 7.53 & 4.04 & 34.00 & 44.99 & 32.70 & 23.63 \\
$\mathrm{~T}$ & 8.38 & 9.75 & 12.17 & 13.58 & 18.15 & 22.12 & 25.46 & 26.15 & 21.99 & 17.31 & 12.84 & 9.44 \\
ETP & 14.91 & 21.53 & 35.05 & 47.03 & 84.35 & 123.39 & 154.63 & 151.32 & 101.62 & 60.30 & 32.20 & 17.87 \\
ETR & 14.91 & 21.53 & 35.05 & 31.85 & 29.51 & 41.92 & 7.53 & 4.04 & 34.00 & 44.99 & 32.20 & 17.87 \\
VR & -2.33 & +5.27 & -9.04 & -0.16 & 0 & 0 & 0 & 0 & 0 & 0 & +0.5 & +5.76 \\
R & 3.93 & 9.2 & 0.16 & 0 & 0 & 0 & 0 & 0 & 0 & 0 & 0.5 & 6.26 \\
DEF & 0 & 0 & 0 & 15.15 & 54.84 & 81.47 & 147.10 & 147.28 & 67.62 & 15.31 & 0 & 0 \\
SUP & 0 & 0 & 0 & 0 & 0 & 0 & 0 & 0 & 0 & 0 & 0 & 0 \\
\hline
\end{tabular}

(FUENTE: Maestre 2000a). P = precipitación media $(\mathrm{mm}) ; \mathrm{T}=$ temperatura media $\left({ }^{\circ} \mathrm{C}\right) ; \mathrm{ETP}=$ evapotranspiración potencial $(\mathrm{mm}) ;$ ETR = evapotranspiración real $(\mathrm{mm}) ; \mathrm{VR}=$ variación de la reserva $(\mathrm{mm}) ; \mathrm{R}=$ reserva de agua en el suelo $(\mathrm{mm}) ; \mathrm{DEF}=$ déficit de agua $(\mathrm{mm}) ; \mathrm{SUP}=$ superávit de agua $(\mathrm{mm})$. 

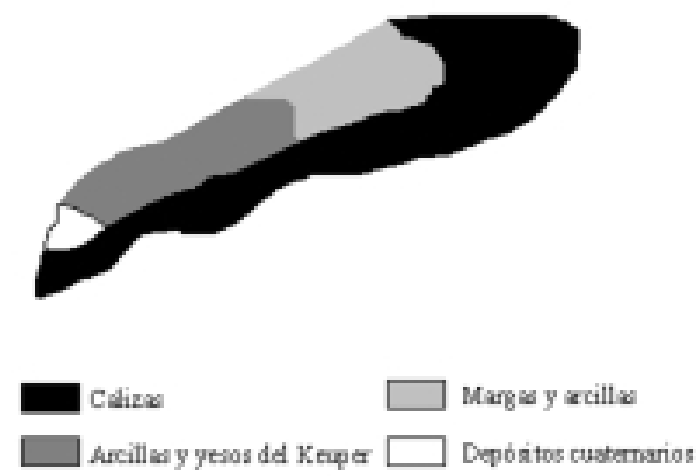

B)

B) $\mathrm{NW}$

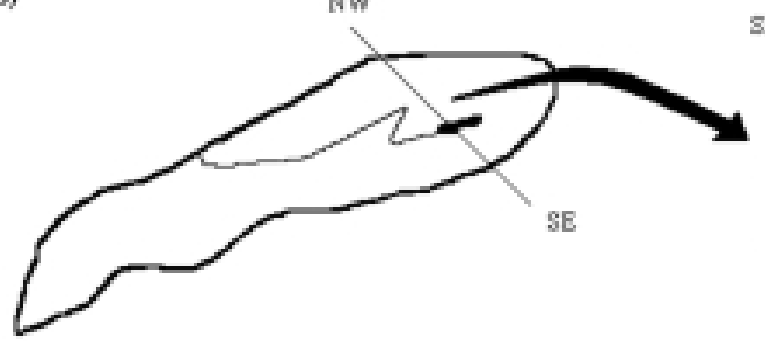

SE

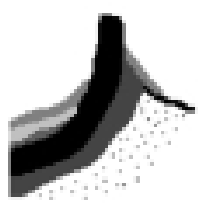

Calizes

Calizas renocas

Depóntos cuaternarios

Calizas marposas

D. Arrilllas grises

Arcillad y yesos del Kenpa

C)
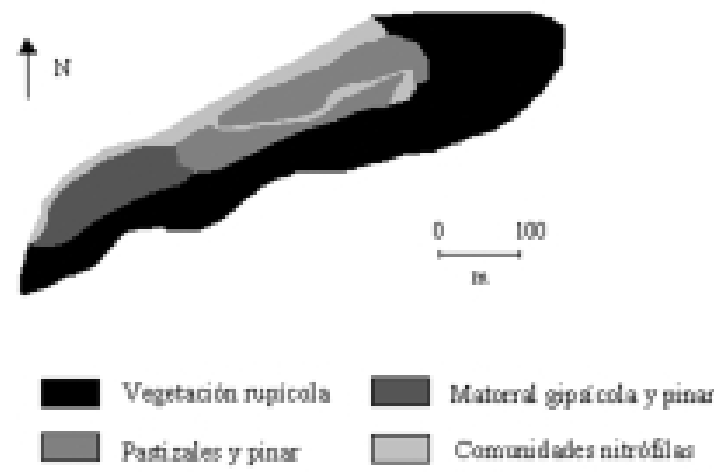

Figura 2. Mapa litológico de la zona de estudio (A), disposición de las litologías en "La Peña" (B) (FUENTE: IGME 1978, Ponce 1987) y distribución de las principales formaciones vegetales (C). 
umbría y la de solana. Estas diferencias microclimáticas van a jugar un importante papel en la distribución de las distintas especies y comunidades vegetales.

En cuanto a los suelos, en toda la zona de roquedo únicamente aparecen Leptosoles líticos, de escaso grosor y perfil A/C, en lugares donde la presencia de grietas posibilita la formación de bolsas térreas. Dentro de la ladera, las intrusiones de material del Keuper en la zona oeste dan lugar a la aparición de un Gipsisol háplico, caracterizado por la presencia de horizontes gípsicos y un epipedión ócrico, mientras que en aquellas zonas donde afloran materiales arcillosos el suelo que se forma es un Regosol (De la Torre y Alías, 1996).

\section{b) Vegetación}

La vegetación presente en la zona de estudio se ve notablemente enriquecida por la gran variedad de ambientes existente, derivada de sus particularidades climáticas, edáficas y geológicas. Un factor importante que ha condicionado el paisaje y la vegetación existente en la actualidad es la repoblación de pino carrasco (Pinus halepensis) realizada en toda la ladera durante los años 50. Las diferentes obras de infraestructura realizadas en su entorno también han influido notablemente en la vegetación, ya que han incrementado las zonas de márgenes y taludes, provocando ambientes propicios para la instalación de comunidades nitrófilas.

Se han tipificado en el área estudiada un total de 23 asociaciones vegetales diferentes, agrupadas en 9 clases y trece órdenes (anexo I). A grandes rasgos, las principales comunidades vegetales que aparecen son las siguientes (figura 2C):

\section{Comunidades rupícolas}

El roquedo es el biotopo más abundante en la zona de estudio, ocupando más del $45 \%$ del total de su superficie (Foto 2). Dentro de su cara norte, las asociaciones más representativas que aparecen son la Rhamno lycioidis-Juniperetum phoeniceae (que agrupa a especies como Juniperus phoenicea, Rhamnus lycioides subsp. lycioides, Rhamnus lycioides subsp. borgiae y Rhamnus alaternus), la Jasonio glutinosae-Teucrietum thymifolii (que incluye, entre otros táxones, a Chiliadenus glutinosus, Hypericum ericoides, Chaenorhinum origanifolium subsp. crassifolium y Teucrium thymifolium) y la Polypodietum serrulati, que presenta como especie más característica a Polypodium cambricum subsp. cambricum y a diversas especies del género Sedum, como $S$. acre y $S$. glanduliferum.

En zonas donde hay acumulaciones de tierra lo suficientemente extensas como para permitir el desarrollo de las especies del matorral se desarrolla la asociación Teucrio homotrichi-Ulicetum dianii, con Ulex parviflorus, Rosmarinus ofricinalis y Sideritis tragoriganum subsp. tragoriganum como especies más representativas. Esta asociación se encuentra fundamentalmente en la zona de umbría, mientras que en la solana estos rellenos son ocupados por espartales de la asociación Helictotricho filifoliae-Stipetum tenacissimae, donde las especies dominantes son Stipa tenacissima y Helictotrichon filifolium, y por matorrales nitrófilos de la asociación Balloto hirsutae-Lavateretum maritimae, formados principalmente por Ballota hirsuta y Lavatera maritima.

2. Formaciones asociadas al pinar

Bajo la cubierta arbórea se desarrollan diversas comunidades herbáceas, siendo la más representativa la asociación Teucrio-Pseudochamaepytis-Brachypodietum ramosi subass. 


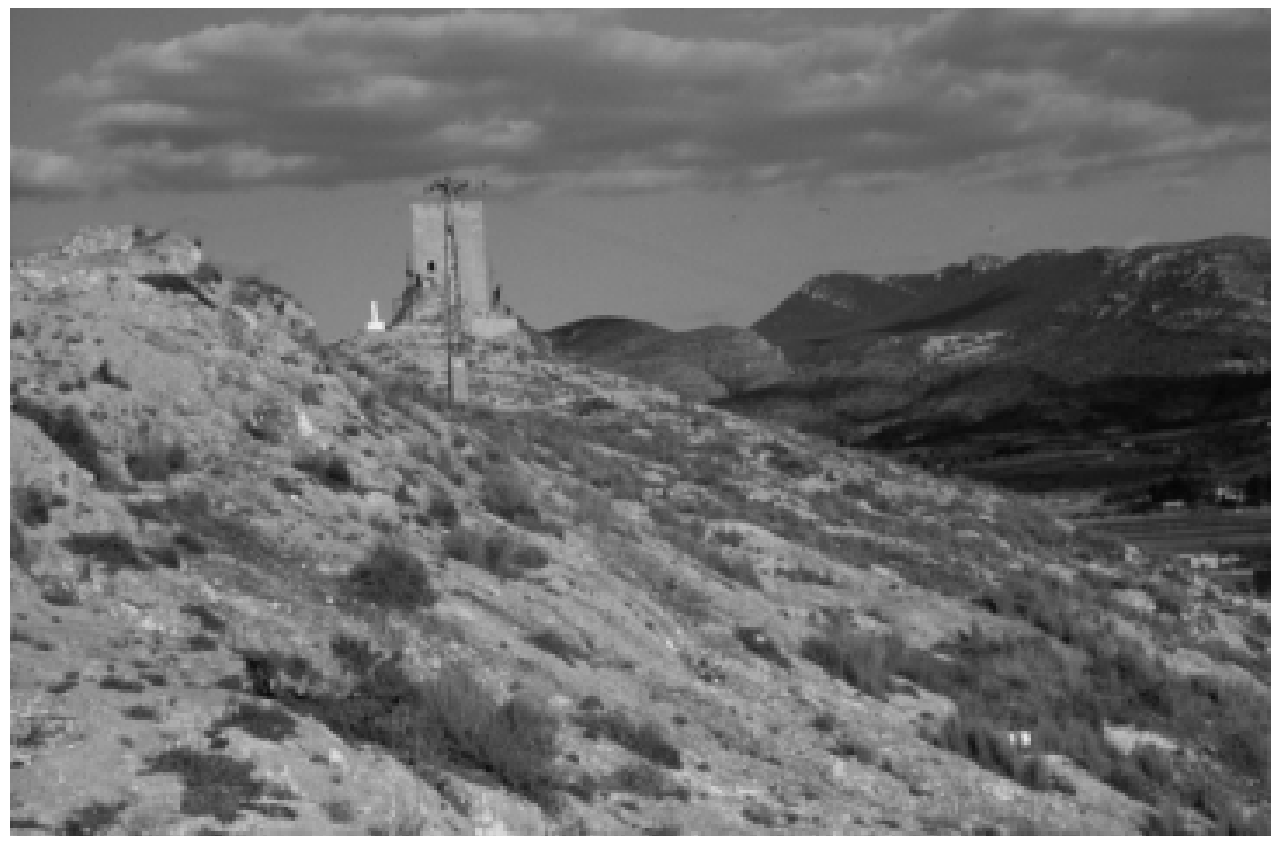

Foто 2. Detalle del roquedo, biotopo más abundante en la zona de estudio.

brachypodietum ramosi, dominada por Brachypodium retusum. La asociación Plantagini albicantis-Stipetum tenacissimae aparece en aquellos claros del pinar donde el suelo ha sido removido, dando lugar a pastizales vivaces que tienen como especie más característica a Stipa parviflora.

En la zona oeste de la ladera, donde la densidad de pinos es menor, aparece la asociación gipsícola Gypsophilo struthii-Teucrietum libanitis, dominada por Ononis tridentata y Helianthemum squamatum, entremezclada con diversas asociaciones halonitrófilas de la Clase Pegano harmalae-Salsoletea vermiculatae como la Salsolo vermiculatae-Artemisietum herba-albae y la Atriplici glaucae-Salsoletum genistoidis.

\section{Comunidades nitrófilas}

Se agrupan dentro de este grupo a numerosas asociaciones vegetales englobadas dentro de las clases Ruderali-Secalietea cerealis, Artemisietea vulgaris y Pegano Harmalae-Salsoletea vermiculatae. Se desarrollan fundamentalmente en aquellas zonas alteradas por la actividad humana, como los bordes de la carretera y del camino de acceso a la fortaleza y aquellos lugares donde se han producido movimientos de tierra. Las asociaciones más representativas que aparecen en la zona de estudio son la Zygophyllo-Atriplicetum glaucae, la Salsolo vermiculatae-Artemisietum herba-albae, la Inulo viscosae-Oryzopsietum miliaceae, la Sisymbrio irionis-Malvetum parviflorae, la Eruco longirostris-Diplotaxietum erucoidis, la Carduo tenuiflori-Hordeetum leporini y la Schismo barbati-Filaginetum congestae. 
La composición florística de la zona de estudio se caracteriza por su diversidad, ya que hasta la fecha se han catalogado un total de 226 táxones agrupados en 56 familias botánicas (Maestre y Pascual, inédito). Al compararla con la de otros territorios que la incluyen se aprecia su riqueza florística, ya que engloba en una superficie inferior a 10 ha el $46 \%$ del total de táxones presentes en el término municipal de Sax -6.300 ha de superficie(Maestre, 1999b) y el 19\% del total de especies catalogadas para el Alto Vinalopó (50.000 ha de superficie) (De la Torre et al., 1997).

El análisis de la flora endémica revela la presencia de 25 especies endémicas (tabla 2), que comprenden el $11 \%$ del total de especies inventariadas. El $80 \%$ pertenecen al grupo de

Tabla 2

PLANTAS ENDÉMICAS PRESENTES EN LA ZONA DE ESTUDIO

\begin{tabular}{|l|c|c|c|}
\hline & & & Categoría \\
Especie & Área de distribución & Hábitat & UICN \\
\hline Avenula murcica & Murcia y Alicante & $\mathrm{P}$ & LRnt \\
Carduus bourgeanus & Iberolevantina & $\mathrm{AN}$ & LRlc \\
Centaurea seridis subsp. cruenta & Iberolevantina & $\mathrm{P}$ & LRlc \\
Chaenorhinum origanifolium subsp. crassifolium & Iberolevantina & $\mathrm{R}$ & LRlc \\
Dianthus broteri subsp valentinus & Iberolevantina & $\mathrm{R}$ & LRlc \\
Diplotaxis harra subsp. lagascana & Iberolevantina & $\mathrm{P}$ & LRlc \\
Fumana ericoides subsp. ericoides & Iberolevantina & $\mathrm{P}$ & LRlc \\
Galium fruticescens & Iberolevantina & $\mathrm{R}$ & LRlc \\
Helianthemum violaceum & Iberolevantina & $\mathrm{MG}$ & LRlc \\
Herniaria fruticosa & Iberolevantina & $\mathrm{MG}$ & LRlc \\
Hypericum ericoides subsp. ericoides & Iberolevantina & $\mathrm{R}$ & LRlc \\
Limonium parvibractaceum & C. Valenciana & $\mathrm{MG}$ & LRnt \\
Ononis tridentata subsp. tridentata & Iberolevantina & $\mathrm{MG}$ & LRlc \\
Onopordum macracanthum subsp. micropterum & Iberolevantina & $\mathrm{AN}$ & LRlc \\
Rhamnus lycioides subsp. borgiae & C. Valenciana y prov. limítrofes & $\mathrm{R}$ & LRlc \\
Rhamnus lycioides subsp. lycioides & Iberolevantina & $\mathrm{R}$ & LRlc \\
Salsola genistoides & Iberolevantina & $\mathrm{MG}$ & LRlc \\
Sarcocapnos saetabensis & Comunidad Valenciana & $\mathrm{R}$ & LRlc \\
Sideritis tragoriganum subsp. tragoriganum & Iberolevantina & $\mathrm{R}$ & LRlc \\
Silene mellifera & Iberolevantina & $\mathrm{R}$ & LRlc \\
Teucrium buxifolium subsp. rivasii & Alicante y Murcia & $\mathrm{R}$ & LRlc \\
Teucrium capitatum subsp. gracillimum & Iberolevantina & $\mathrm{P}$ & LRlc \\
Teucrium gnaphalodes & Iberolevantina & $\mathrm{P}$ & LRlc \\
Teucrium murcicum subsp. murcicum & Iberolevantina & $\mathrm{R}$ & LRlc \\
Teucrium thymifolium & Iberolevantina & $\mathrm{R}$ & LRlc \\
\hline
\end{tabular}

(FUENTE: Maestre 1999c). Área de distribución y categorías UICN según Laguna et al. (1998) y Moreno y Sainz (1992). $\mathrm{P}=$ pastizal, $\mathrm{AN}=$ Ambientes nitrificados, $\mathrm{R}=$ roquedo, $\mathrm{MG}=$ matorral gipsícola, $\mathrm{LRlc}=$ preocupación menor y LRnt $=$ casi amenazada. 
los endemismos iberolevantinos, aunque también aparecen especies exclusivas de la Comunidad Valenciana y provincias limítrofes. Cuando se analizan los biotopos que ocupan estas especies se aprecia la importancia del roquedo, refugio del $48 \%$ de endemismos. Las zonas de pastizal y los matorrales gipsícolas, con un 24 y $20 \%$ del total de táxones endémicos son los siguientes ambientes en importancia. Por lo que respecta al grado de amenaza según las categorías de la Unión Internacional para la Conservación de la Naturaleza (IUCN, 1994) establecidas para la flora endémica de la Comunidad Valenciana (Laguna et al., 1998), se observa como la práctica totalidad de los endemismos se encuadran dentro de la categoría de «preocupación menor», lo que indica que no están amenazadas dentro su territorio. La importancia de la flora endémica en la zona queda reflejada al comparar su densidad con la de otros territorios cercanos inventariados (tabla 3). Las reducidas dimensiones que presenta hace que esta densidad de endemismos sea anormalmente elevada, superando a otros territorios de gran riqueza florística, como el Valle de Villena, las comarcas de La Marina y el propio término municipal de Sax.

Tabla 3

RESUMEN FLORÍSTICO DE LA ZONA DE ESTUDIO Y DE OTRAS ÁREAS DE ALICANTE INVENTARIADAS

\begin{tabular}{|l|c|c|c|c|c|c|}
\hline Área & S & T & E & F & DE & Referencia \\
\hline La Marina Alta & 736 & 1470 & 102 & 6.94 & 0.14 & Pérez Badía (1997) \\
La Marina Baja & 590 & 1304 & 112 & 8.59 & 0.19 & Solanas (1998) \\
Alto Vinalopó & 500 & 1182 & 101 & 8.54 & 0.20 & De la Torre et al. (1997) \\
Valle de Villena & 94 & 697 & 52 & 7.46 & 0.55 & Alonso (1996) \\
Sax & 63 & 488 & 42 & 8.61 & 0.67 & Maestre (1999b) \\
Zona de estudio & 0.1 & 226 & 25 & 11.06 & 250 & Este estudio \\
\hline
\end{tabular}

$\mathrm{S}=$ Superficie $\left(\mathrm{km}^{2}\right), \mathrm{T}=$ número de táxones, $\mathrm{E}=$ número de endemismos, $\mathrm{F}=$ porcentaje de flora endémica $\mathrm{y}$ $\mathrm{DE}=$ densidad de endemismos (número de endemismos $/ \mathrm{km}^{2}$ ).

Por lo que respecta a la flora protegida, aparecen 10 especies incluidas dentro del Anexo III de la Orden de 20 de diciembre de 1985 de la Conselleria de Agricultura y Pesca de la Generalitat Valenciana (Chiliadenus glutinosus, Hypericum ericoides subsp. ericoides, Micromeria fruticosa, Teucrium buxifolium subsp. rivasii, T. capitatum subsp. gracillimum, T. gnaphalodes, T. murcicum subsp. murcicum, T. pseudochamaepytis, T. thymifolium y Thymus vulgaris subsp. vulgaris), que supedita su tala o desenraizamiento a una autorización previa por parte de este organismo.

\section{d) Hábitats de interés comunitario}

Se han encontrado en la zona estudiada un total de 9 comunidades vegetales de interés comunitario, de los que 4 (el 44\% del total) son de conservación prioritaria (tabla 4). Si se analizan las apetencias ecológicas de estos sintáxones se observa que la mayoría (el $44 \%$ del total) corresponden a matorrales halonitrófilos y a formaciones rupícolas. Comparando el número de hábitats con el de otros territorios que la incluyen, en el área de estudio aparecen el $41 \%$ del total presente en el término municipal de Sax (Maestre, 2000b) y el 20\% del total existente en la Comarca del Alto Vinalopó (De la Torre et al., 1997). 


\begin{tabular}{|l|c|}
\hline Hábitat & $\begin{array}{c}\text { Ambiente que ocupa } \\
\text { en la zona de estudio }\end{array}$ \\
\hline Atriplici glaucae-Salsoletum genistoidis & $\mathrm{M}$ \\
Comunidad de Ononis tridentata $*$ & $\mathrm{M}$ \\
Gypsophilo struthii-Teucrietum libanitidis * & $\mathrm{M}$ \\
Jasonio glutinosae-Teucrietum thymifolii & $\mathrm{R}$ \\
Polypodietum serrulati & $\mathrm{R}$ \\
Rhamno lycioidis-Juniperetum phoeniceae subass. rhamnetosum borgiae * & $\mathrm{R}$ \\
Salsolo vermiculatae-Artemisietum herba-albae & $\mathrm{M}$ \\
Sedetum micrantho-sediformis subass. sedetosum glanduliferi & $\mathrm{R}$ \\
Teucrio pseudochamaepitys-Brachypodietum ramosi subass. & \\
Brachypodietosum ramosi $*$ & $\mathrm{P}$ \\
\hline
\end{tabular}

$\mathrm{M}=$ matorral, $\mathrm{R}=$ roquedo $\mathrm{y} \mathrm{P}=$ pastizal.

\section{e) Fauna}

Las reducidas dimensiones de la zona de estudio y su cercanía al casco urbano no permiten la presencia de numerosas especies animales. No obstante, se han encontrado un total de 21 especies de vertebrados (tabla 5), de los que 10 están catalogadas de «interés especial» por el Catálogo Nacional de Especies Amenazadas (R. D. 439/1990, actualizado por las órdenes de 29 de agosto de 1996, 9 de julio de 1998 y 9 de junio de 1999).

Tabla 5

VERTEBRADOS ENCONTRADOS EN LA ZONA DE ESTUDIO. CON * SE SEÑALAN LAS ESPECIES DE “INTERÉS ESPECIAL” SEGÚN EL CATÁLOGO NACIONAL DE ESPECIES AMENAZADAS

\begin{tabular}{|c|c|}
\hline Aves & Reptiles \\
\hline Gorrión común (Passer domesticus) & Lagartija común (Podarcis hispanica) * \\
\hline Vencejo real (Apus melba)* & Lagartija colilarga (Psammodromus algirus) ${ }^{*}$ \\
\hline Vencejo común (Apus apus) * & Salamanquesa común (Tarentola mauritanica) * \\
\hline Golondrina común (Hirundo rustica) * & Salamanquesa rosada (Hemidactylus turcicus) * \\
\hline Avión común (Delichon urbica) ${ }^{*}$ & Mamíferos \\
\hline Mirlo común (Turdus merula) & Rata común (Rattus norvegicus) \\
\hline Cernícalo vulgar (Falco tinnunculus) * & Ratón común (Mus musculus) \\
\hline Estornino negro (Sturnus unicolor) & Murciélago común (Pipistrellus pipistrellus) * \\
\hline Verdecillo (Serinus serinus) & Erizo común (Erinaceus europaeus) \\
\hline Verderón (Charduelis chloris) & Conejo (Oryctolagus cunniculus) \\
\hline Cuervo (Corvus corax) & Liebre (Lepus capensis) \\
\hline
\end{tabular}


El peligro de desprendimientos en la zona de estudio ha sido objeto de preocupación por parte de los vecinos y autoridades desde hace varias décadas, apareciendo los primeros estudios serios sobre este tipo de riesgo a finales de los años 80. La cercanía del casco urbano al roquedo y los desprendimientos acaecidos en la última década hace que exista un elevado grado de peligrosidad que ha sido puesta de manifiesto en trabajos anteriores (Marco y Matarredona, 1993; Maestre, 2000a). En 1990 se produjo el desprendimiento de un bloque rocoso de unas $3 \mathrm{Tm}$ sobre el casco urbano que provocó daños materiales en diversas viviendas. Ante este episodio se aceleraron las obras de consolidación del macizo rocoso, que finalmente se realizaron durante el año 1991. Estos trabajos consistieron en un «grapado» de las rocas e inyección de hormigón en las juntas de las rocas más abiertas, así como en la colocación de unos pivotes de protección en los lugares más sensibles, instalándose también un vallado y muro de seguridad en la cara sur del roquedo (figura $3 \mathrm{~A}$ ).

En cuanto a la erosión, se localizan procesos erosivos en lugares muy concretos, como ocurre en los taludes del camino de acceso, de la carretera que bordea la ladera y en algunos puntos del interior del pinar (figura 3B). En los taludes aparecen pequeños surcos a consecuencia de la falta de la vegetación, mientras que en el interior del pinar aparecen algunas cárcavas de mayor tamaño en la zona de umbría.

\section{g) Recursos culturales}

Entre los recursos histórico-artísticos presentes en la zona de estudio destaca el Castillo de Sax, fortaleza roquera de plana alargada y carácter irregular que se adapta al escarpado peñasco sobre el que se sustenta (foto 3). Este castillo tiene en su extremo una torre

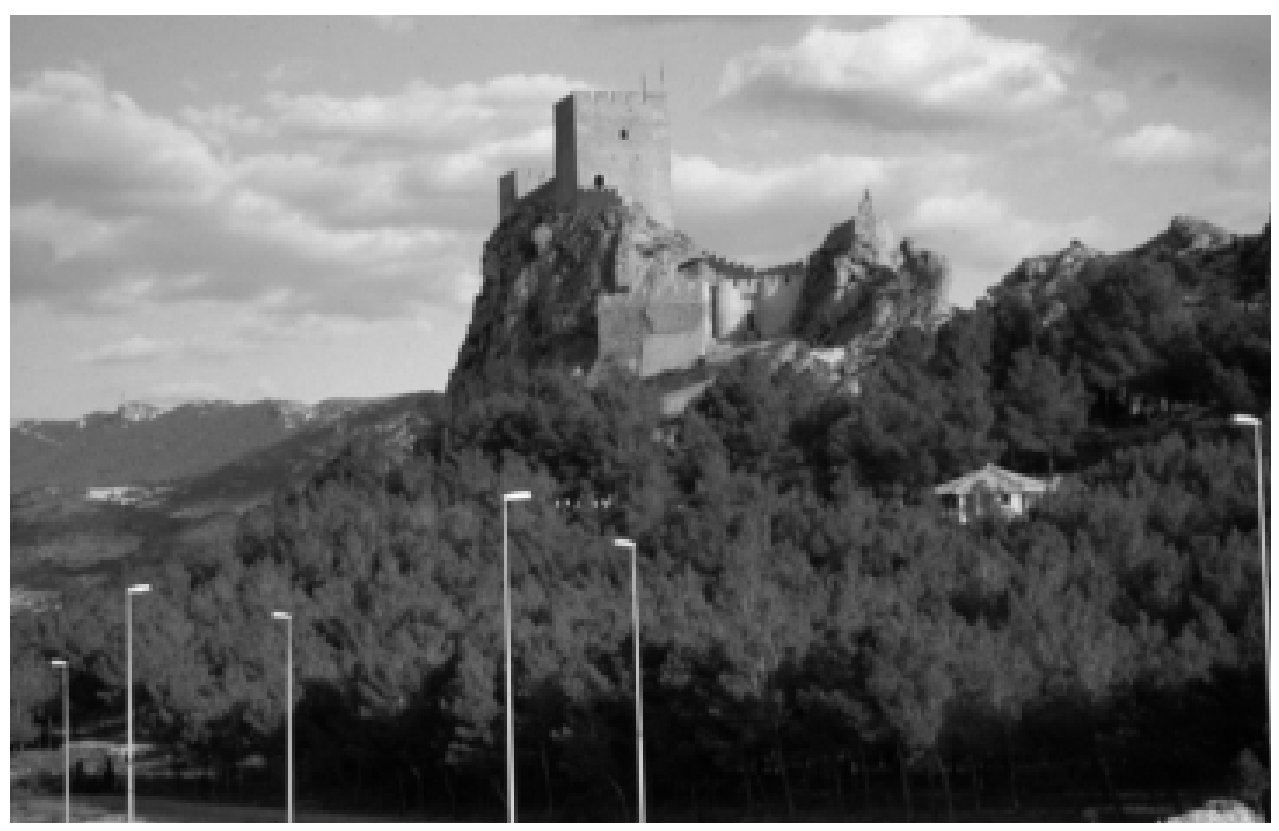

Fото 3. Castillo de Sax y pinar en la cara norte de la zona de estudio. 
cuadrada de tapial y de una sola planta, de época almohade (s. XII), y en su parte suroeste, otra torre cuadrada de mayor tamaño (la Torre del Homenaje) construida a mediados del Siglo XIV. Entre estas dos torres se encuentra un aljibe cubierto con bóveda de medio cañón (Azuar y Ruibal, 1998). Otro recurso histórico de interés es el «Pocico de la nieve», nevero presente en la ladera norte construido a principios del siglo XVIII (Vázquez, 1996).

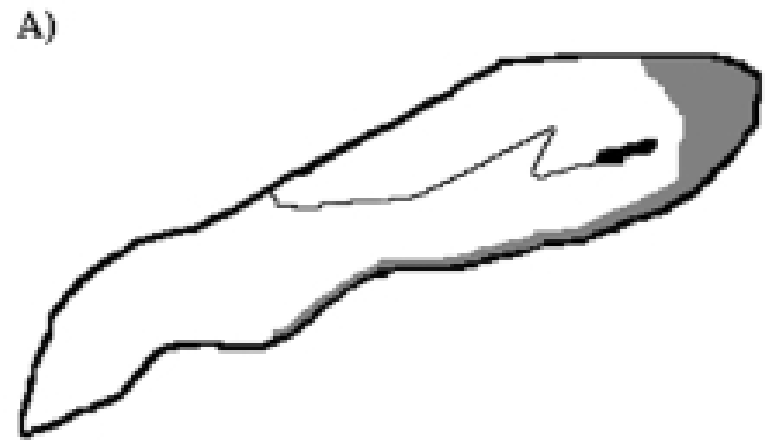

B)

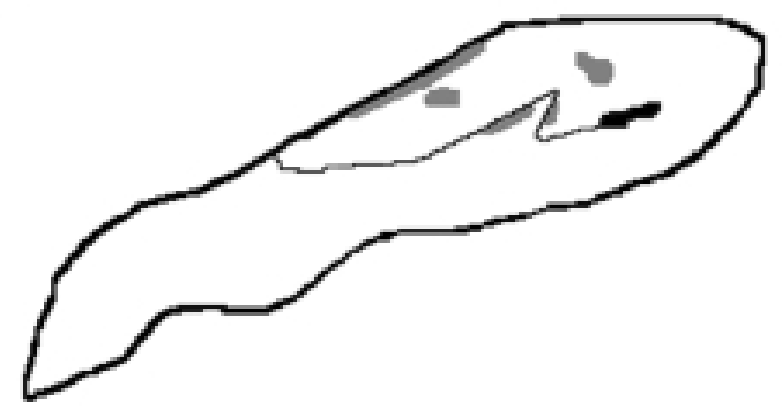

C)

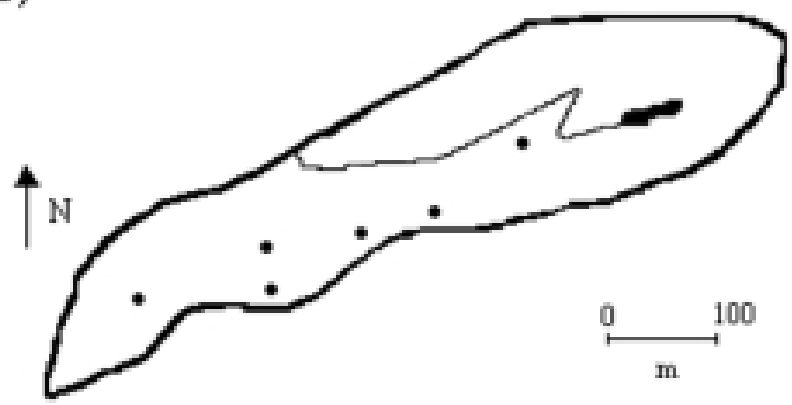

FIGURA 3. Lugares donde se ha actuado contra los desprendimientos (A), donde se han detectado procesos erosivos (B) y distribución de los principales vertederos ilegales (C). 
Entre los recursos arqueológicos, hay que destacar la presencia en la falda norte del Castillo de yacimientos arqueológicos pertenecientes a la Edad del Bronce (II milenio a. C.) y a las épocas ibérica, romana y medieval. Entre todos los restos encontrados destacan los vestigios de un asentamiento de la Edad del Bronce que forma parte del núcleo habitado más antiguo que se ha encontrado en el municipio (Pérez Burgos, 1998).

Dentro de los recursos etnológicos hay que mencionar el importante papel que juega el Castillo durante la celebración de los días de Pascua en Sax, ya que es costumbre entre los habitantes de este municipio el visitar este paraje. Desde el punto de vista científico-educativo, su riqueza florística y de comunidades vegetales, su cercanía al casco urbano y su accesibilidad le convierten en un lugar ideal para la realización de estudios científicos y actividades de educación ambiental.

\section{Principales impactos ambientales}

\section{a) Vertederos ilegales}

La presencia de vertederos incontrolados constituye uno de los principales impactos paisajísticos y ambientales existentes la zona estudiada (figura 3C). En la cara sur del roquedo, lindando con el casco urbano, aparecen las mayores acumulaciones de residuos sólidos formadas principalmente por basura doméstica, muebles, escombros y electrodomésticos. Por lo que respecta a la zona norte, existen dos pequeños vertederos de una dimensión aproximada de $150 \mathrm{~m}^{2}$ donde se depositan fundamentalmente basura doméstica, muebles y residuos industriales como colas y disolventes (foto 4).



Fото 4. Vertedero ilegal de residuos sólidos junto al roquedo. 
En ocasiones estos vertederos se queman intencionadamente con el fin de deshacerse de los residuos allí depositados. Esta acción entraña un serio peligro de incendio forestal en el paraje, debido a la densidad de pinos existente y a la abundancia de especies herbáceas y de matorral altamente pirofíticas como la aliaga (Ulex parviflorus), el romero (Rosmarinus officinalis) y el labiérnago (Phyllirea angustifolia) (Vélez, 1990).

\section{b) Obras de infraestructura}

Entre el impacto provocado por las diversas obras de infraestructura realizadas en el entorno del Castillo hay que destacar los daños causados por los trabajos de consolidación del roquedo, la ampliación del camino de acceso y de la carretera que bordea a la ladera y la instalación del nuevo alumbrado de la fortaleza, así como los recientes aterrazamientos realizados en la parte oeste del paraje.

Las obras de consolidación del macizo rocoso alteraron el hábitat de diversas comunidades rupícolas, como las asociaciones Jasonio glutinosae-Teucrietum thymifolii y Polypodietum serrulati, causando una disminución considerable de los efectivos de Polypodium cambricum subsp. cambricum, Chaenorhinum origanifolium subsp. crassifolium, Teucrium buxifolium subsp. rivasii y T. thymifolium, especies cuya área de distribución municipal se reduce a este paraje (Maestre, 1999b).

La ampliación del camino de acceso y de la carretera que bordea la ladera —obras realizadas en 1998 y 1994 respectivamente- no han provocado un impacto muy importante al no afectar a las formaciones rupícolas y gipsícolas. No obstante, han propiciado la aparición de procesos erosivos en los taludes creados y favorecido la proliferación de especies nitrófilas como resultado de los movimientos de tierra.

La instalación del nuevo alumbrado del Castillo fue realizada en 1997 bajo los auspicios del Instituto Valenciano de Turismo. Los efectos sobre las comunidades vegetales no han sido muy perjudiciales debido a que la mayoría de los focos se sitúan sobre la base de la fortaleza. Tan sólo la instalación de algunas torres con focos y la colocación de tuberías subterráneas para albergar la instalación eléctrica ha provocado la alteración de determinadas zonas en el interior del pinar, habiendo afectado fundamentalmente a la asociación Teucrio-Brachypodietum ramosi subass. brachyepodietum ramosi, que ha sido sustituida por comunidades nitrófilas de la Clase Ruderali-Secalietea Cerealis.

La última actuación efectuada en su entorno ha sido el aterrazamiento de parte de la ladera en la zona este para realizar una repoblación de pino carrasco (Pinus halepensis). Estas obras, realizadas durante el mes de enero de 2000 con maquinaria pesada, han eliminando la vegetación preexistente, afectando negativamente a la vegetación gipsícola de esta parte del paraje (foto 5). Entre las especies y comunidades vegetales afectadas se encuentran endemismos como Herniaria fruticosa y Helianthemum violaceum, así como hábitats de interés comunitario como la asociación Gypsophilo struthiii-Teucrietum libanitidis y la comunidad de Ononis tridentata.

\section{Legislación aplicable}

La ley 11/1994, de 27 de diciembre, de Espacios Naturales Protegidos de la Comunidad Valenciana entró en vigor el 9 de enero de 1995. En su capítulo II, dentro del Título I, se recoge el régimen general de Espacios Naturales Protegidos, que contempla la creación de siete figuras de protección (Parques naturales, Parajes naturales, Parajes naturales 




Fото 5. Movimientos de tierra para plantar pinos en la zona este de la ladera.

municipales, Reservas naturales, Monumentos naturales, Sitios de interés y Paisajes protegidos).

Como herramientas de ordenación y gestión de estos ENP, este documento establece cuatro instrumentos básicos: planes de ordenación de los recursos naturales (P.O.R.N.), planes rectores de uso y gestión (P. R.U.G.), planes especiales y normas de protección. Los P.O.R.N. son requisito previo para la ordenación de parques y reservas naturales. Los P.R.U.G. regularán la ordenación de los parques y reservas naturales, siendo también necesaria su aprobación en el caso de parajes naturales y paisajes protegidos. Los planes especiales regulan la protección de parajes naturales municipales, mientras que las normas de protección rigen la ordenación de los monumentos naturales y sitios de interés.

Entrando a valorar la posible aplicación de alguna figura de protección a la zona de estudio, las categorías de parque y reserva natural no son muy apropiadas por las reducidas dimensiones que ésta presenta y su problemática ambiental, ya que la ausencia de conflictos urbanísticos y de usos actuales hace innecesarios la elaboración de los correspondientes P.O.R.N. y P.R.U.G. Además, estas categorías suelen aplicarse a espacios naturales de especial importancia autonómica o nacional susceptibles de ser incluidos en redes internacionales de ENP. Estos factores, unidos a que es el Gobierno Valenciano el encargado de proponer su declaración, hacen que no se considere su aplicación a la zona de estudio.

La figura de paraje natural también requiere la elaboración de un P.R.U.G., por lo que tampoco es apropiada. Las categorías de monumento natural y sitio de interés son más 
acordes a las características del paraje estudiado y podrían aplicarse satisfactoriamente. No obstante, el hecho de que la iniciativa de su declaración corra a cargo de la Conselleria de Medio Ambiente y la necesidad de elaborar normas de protección dificulta su posible declaración.

El elevado interés local que tiene el Castillo y su entorno, la condición de elaborar planes especiales de protección cuyo único requisito legal es que respeten la legislación urbanística y el hecho de que sea el propio ayuntamiento el encargado de proponer al Gobierno Valenciano su declaración — con la consiguiente agilización de trámites burocráticos- y asumir su manejo hacen que la figura de paraje natural municipal sea la que mejor se ajusta a sus características.

Otra figura alternativa que podría aplicarse al margen de las recogidas en la ley 11/1994 es la de microrreserva vegetal, creada mediante el decreto 218/1994, de 17 de octubre, del Gobierno Valenciano. Esta categoría jurídica fue creada para conservar a las distintas especies endémicas existentes en la Comunidad Valenciana, que suelen seguir unos patrones de microdistribución coincidentes con la aparición de suelos azonales e intrazonales (Laguna, 1995). Por lo tanto, surge como una nueva figura compatible y complementaria dentro de los ENP, pero con interesantes peculiaridades en sus fines, ya que tiene como objetivos fundamentales el fomento de la conservación, la educación ambiental y la investigación científica (Padilla y Ramón, 1997; Laguna et al., 1998). El proceso legislativo para su declaración corre a cargo del Gobierno Valenciano, pudiendo también otras entidades públicas o particulares solicitar la declaración.

La diversidad florística y la elevada densidad de plantas endémicas de la zona de estudio podrían propiciar su declaración como microrreserva vegetal. No obstante, hay que tener en cuenta el hecho de que los endemismos presentes en el Castillo y su entorno estén ya incluidos en alguna de las microrreservas existentes en la provincia de Alicante. Con el fin de estudiar la idoneidad de la implantación de una microrreserva son necesarios estudios más detallados sobre su flora y la del resto de microrreservas, por lo que no se considera en este trabajo la aplicación de esta figura legal.

\section{Consideraciones finales}

El Castillo de Sax y su entorno cumple todos los requisitos para su declaración como paraje natural municipal. Desde el punto de vista urbanístico y de la propiedad, su calificación como suelo no urbanizable de protección especial por las normas subsidiarias vigentes (VV.AA., 1997), su pertenencia municipal y la ausencia de usos conflictivos en su territorio facilitan la elaboración de las normas de protección y deja vía libre a la corporación municipal para proponer su declaración.

El interés ecológico en el ámbito local y comarcal de la zona de estudio ha quedado puesto de manifiesto tras el análisis de su medio físico, ya que no sólo alberga una gran riqueza florística y una densidad de especies endémicas inusual, sino que además la propia formación geológica es notablemente singular por su aspecto y características. Junto a este interés ecológico hay que destacar su importante valor histórico, turístico y cultural debido a la presencia del Castillo y a otros recursos culturales, así como las posibilidades que ofrece para la realización en su entorno de actividades científicas, de educación ambiental y de turismo rural. Las continuas agresiones que viene sufriendo hacen necesaria su declaración como zona protegida, con el fin de preservar la riqueza biológica y cultural que encierra. 


\section{Agradecimientos}

Durante la toma de datos de campo contamos con la inestimable colaboración de $\mathrm{M}^{\mathrm{a}}$ Dolores Puche y Mónica Calabuig.

\section{Bibliografía}

ALCARAZ, F., SÁNCHEZ-GÓMEZ, P. y DE LA TORRE, A. (1991): Datos sobre la vegetación de Murcia. Diego Marín Editor. Murcia.

ALONSO, M ${ }^{a}$ A. (1996): Flora y Vegetación del Valle de Villena (Alicante). Instituto de Cultura Juan Gil-Albert y Generalitat Valenciana. Alicante.

AZUAR, R. y RUIBAL, A. (1998): Castillos de Alicante y Albacete. Bancaja. León.

BRAUN-BLANQUET, J. (1978): Fitosociología. Editorial Blume. Madrid.

DE LA TORRE, A. y ALÍAS, L. (1996): Suelos y vegetación en el Alto Vinalopó. Servicio de Publicaciones de la Universidad de Alicante. Alicante.

DE LA TORRE, A., ALCARAZ, F. y VICEDO, M. (1997): El Paisaje Vegetal del Alto Vinalopó (Alicante). Universidad de Alicante. Edición electrónica. Alicante.

FAO-UNESCO. (1988): Soil Map of the World. Revised Legend. World Resources Report 60. FAO. Roma.

GARROD, G. D. y WILLIS, K. G. (1994): «Valuing biodiversity and nature conservation at a local level». Biodiversity and Conservation, 3, pp. 555-565.

GENERALITAT VALENCIANA. (1996): Legislación medioambiental de la Comunidad Valenciana. Conselleria de Agricultura y Medio Ambiente. Valencia.

IGME. (1978): Mapa geológico de España, escala 1:50000. Hoja 846 (Castalla). Ministerio de Industria y Energía. Madrid.

IUCN. (1994): Categorías de las Listas Rojas de la UICN, preparadas por la Comisión de Supervivencia de Especies de la UICN. International Union for Conservation of Nature and Natural Resources. Gland.

LAGUNA, E. (1995): «Microrreservas de flora: un nuevo modelo de conservación en la Comunidad Valenciana». Quercus, 118, pp. 22-26.

LAGUNA, E., CRESPO, M. B., MATEO, G., LÓPEZ, S., FABREGAT, C., SERRA, L., HERRERO-BORGOÑÓN, J. L., AGUILELLA, A. y FIGUEROLA, R. (1998): Flora endémica, rara o amenazada de la Comunidad Valenciana. Generalitat Valenciana, Valencia.

LLORENS, V. y RODRÍGUEZ, J. (1991): Els espais naturals protegits a Espanya. Ediciones Alfonso el Magnánimo e Institución Valenciana de Estudios e Investigación. Valencia.

KEEN, M. y MERCER, D. (1993): «Environmental planning at the local level: The example of local conservation strategies in Victoria, Australia». Environmentalist, 13, pp. 83-95.

KENNY-GILDAY, C., BUZZELLI, D. MCDONALD, D., LOW, B. y CONTE, L. (1995): «The question of sustainable development». Nature Conservancy, 45, pp. 10-15.

MAESTRE, F.T. (1998a): «Notas sobre la riqueza biológica del Castillo de Sax». Revista de Fiestas de Moros y Cristianos, pp.154-156. Mayordomía de San Blas. Sax.

MAESTRE, F.T. (1998b): «Contribución al conocimiento de la flora y vegetación de Sax (Alicante)». Revista del Vinalopó, 1, pp. 105-118.

MAESTRE, F.T. (1999a): «Apuntes sobre la conservación y gestión de los espacios naturales de Sax». Revista del Vinalopó, 2, pp. 119-138. 
MAESTRE, F.T. (1999b): «Flora vascular del término municipal de Sax (Alicante)». Ecología, 13, pp. 45-86.

MAESTRE, F.T. (1999c): «Notas sobre la flora vascular endémica de Sax (Alicante)». Blancoana, 16, pp. 3-21.

MAESTRE, F.T. (2000a): El medio ambiente en Sax. Estado actual y propuestas de gestión. Universidad de Alicante y Ayuntamiento de Sax. Alicante (en prensa).

MAESTRE, F.T. (2000b.): «Listado de hábitats de interés comunitario del término municipal de Sax (Alicante, SE España)». Boletín de la Real Sociedad Española de Historia Natural (Sección Biología), 96, pp. 193-203.

MARCO, J. A. y MATARREDONA, E. (1993): «Aproximación geográfica al tema de los riesgos en la provincia de Alicante». Canelobre, 27, pp. 59-66.

MATEO, G. y CRESPO, M. B. (1998): Manual para la determinación de la Flora Valenciana. Monografías de flora Montibérica ${ }^{\circ} 3$. Alicante-Valencia.

MORENO, J.C. y SAINZ, H. (1992): Atlas corológico de las monocotiledóneas endémicas de la Península Ibérica e Islas Baleares. Icona. Madrid.

MULERO, A. (1999): Introducción al medio ambiente en España. Ariel Geografía. Barcelona.

PADILLA, A. y RAMÓN, A. (1997): «Planeamiento ambiental a escala de detalle: microrreservas de flora en la Comunidad Valenciana». Investigaciones Geográficas, 17, pp. 117-128.

PÉREZ BADÍA, M. R. (1997): Flora vascular y vegetación de la comarca de la Marina Alta. Instituto de Cultura Juan Gil Albert. Alicante.

PÉREZ BURGOS, J.M. (1998): «Arqueología de Sax». El Castillo de Sax, 5, pp. 12-14.

PONCE, G. (1987): «Breves notas sobre el origen y la formación geológica de la Peña». Revista de Fiestas de Moros y Cristianos. Mayordomía de San Blas. Sax.

SECRETARIA GENERAL DE MEDIO AMBIENTE. (1996): Guía para la elaboración de estudios del medio físico. Contenido y metodología. Ministerio de Medio Ambiente. Madrid.

SOLANAS, J.L. (1998): Flora, Vegetació i Fitogeografia de La Marina Baixa. Universidad de Alicante. Edición electrónica. Alicante.

VV.AA. (1997): Revisión de normas subsidiarias de planeamiento. Informe inédito. Ayuntamiento de Sax. Sax.

VÁZQUEZ, V. (1996): Sax, ayer y hoy en la plumilla de Pedro Estevan. Mayordomía de San Blas. Sax.

VÉLEZ, I. (1990): «Algunas observaciones para una selvicultura preventiva de incendios forestales». Ecología, fuera de serie n ${ }^{\circ}$ 1, pp. 561-571. 


\section{ANEXO I}

\section{Esquema sintaxonómico de la zona de estudio}

\section{Clase QUERCETEA ILICIS BRAUN-BLANQ. 1947}

Ord. Pistacio lentisci-Rhamnetalia alaterni Rivas-Mart. 1975

All. Rhamno lycioidis-Quercion cocciferae Rivas Goday ex Rivas-Mart. 1975

Ass. Rhamno lycioidis-Juniperetum phoeniceae Rivas Mart. et López González in López González 1976 subass. rhamnetosum borgiae De la Torre et Alcaraz inéd.

\section{Clase LYGEO SPARTI-STIPETEA TENACISSIMAE RIVAS MART. 1978}

Ord. Lygeo-Stipetalia tenacissimae Braun-Blanq. et O. Bolòs 1958 em Rivas Mart. 1978.

All. Stipion tenacissimae Rivas Mart. 1978

Ass. Helictotricho filifoliae-Stipetum tenacissimae Costa, Peris et Stübing. 1988

All. Thero-Brachypodion ramosi Braun-Blanq. 1925

Ass. Teucrio-Brachypodietum ramosi O. Bolòs 1957

subass. brachypodietosum ramosi

Ord. Hyparrhenietalia hirtae Rivas Mart. 1978

All. Stipion parviflorae De la Torre et Alcaraz inéd.

Ass. Plantagini albicantis-Stipetum parviflorae De la Torre et Alcaraz inéd.

All. Saturejo-Hyparrhenion hirtae O. Bolòs 1962

Ass. Aristido coerulescentis-Hyparrhenium pubescentis Rivas-Martínez et Alcaraz in

Alcaraz 1988 subass. avenuletosum murcicae.

3. Clase ROSMARINETEA OFFICINALIS BRAUN-BLANQ. 1947 EM. RIVAS MART. ET AL. 1991

Ord. Rosmarinetalia officinalis Braun-Blanq. 1931

All. Rosmarino-Ericion multiflorae Braun- Blanq. 1931

Suball. Teucrium latifoliae-Thymenion piperellae Stubing, Peris et Costa 1989

Ass. Teucrio homotrichi-UIicetum dianii Alcaraz et De la Torre 1988

Ord. Gypsophiletalia (Bellot 1952) Bellot et Rivas Goday in Rivas Goday 1956

All. Lepidion subulati (Bellot 1952) Bellot et Rivas Goday in Rivas Goday 1956

Ass. Comunidad de Ononis tridentata

Suball. Thymo ciliati-Teucrienion verticillati (Rivas Goday 1956) Alcaraz,

Sánchez-Gómez, De la Torre, Ríos et Rogel. 1991

Ass. Gypsophilo struthii-Teucrietum libanitis Rivas Goday et Rigual 1956 corr.

Rivas Goday et Rivas Mart. 1969

\section{Clase PEGANO HARMALAE-SALSOLETEA VERMICULATAE BRAUN-BLANQ. ET O. BOLÒS 1958}

Ord. Salsolo vermiculatae-Peganetalia harmalae Braun-Blanq. et O. Bolòs 1954

All. Salsolo vermiculatae-Peganion harmalae Braun-Blanq. et O. Bolòs 1954 em. O. Bolòs 1967

Ass. Salsolo vermiculatae-Artemisietum herba-albae (Braun-Blanq. et O. Bolòs 1958)

O. Bolòs 1967

Ass. Salvio-Plantaginetum albicantis (O. Bolòs et R. Mol.) O. Bolòs 1983

Ass. Balloto hirsutae-Lavateretum maritimae Cantó, Laorga et Belmonte 1986

All. Haloxylo-Atriplicion glaucae Rivas Goday 1963

Ass. Atriplici glaucae-Salsoletum genistoidis O. Bolòs (1957) 1967 
5. Clase ARTEMISIETEA VULGARIS LOHMEYER, PRESING ET R. TUXEN 1950

Subcl. Onopordenea acanthii Rivas Mart., Báscones, Díaz, Fernández Gonzalez et Loidi 1991

Ord. Carthametalia lanati Brullo in Brullo et Marceno 1985

All. Onopordion nervosi Braun-Blanq. et O. Bolòs 1958 corr. Rivas Mart. 1975

Suball. Onopordenion nervosi

Ass. Carthamo lanati-Picnomonetum acarnae M.B. Crespo 1989

All. Bromo-Oryzopsion miliacei O. Bolòs 1970.

Ass. Inulo viscosae-Oryzopsietum miliaceae A. et O. Bolòs in O. Bolòs 1957

Ass. Zygophyllo-Atriplicetum glaucae Rivas Goday, Esteve et Rigual 1958

6. Clase RUDERALI-SECALIETEA CEREALIS BRAUN-BLANQ. 1936

Subcl. Chenopodienea muralis Rivas Mart., Báscones, Díaz, Fernádez González et Loidi 1991

Ord. Sisymbrietalia officinalis J. Tüxen in Lohmeyer et al. 1962 em. Rivas Mart. et al. 1991

Subord. Sisymbrienalia officinalis

All. Hordeion leporini (Braun-Blanq. 1931) Braun-Blanq. 1947

Ass. Carduo tenuiflori-Hordeetum leporini Braun-Blanq. 1936

Ass.Eruco longirrostris-Diplotaxietum erucoidis Rigual 1972 corr. Alcaraz 1984 subass. senecietosum gallici

Ass. Schismo barbati-Filaginetum congestae O. Bolòs (1948) 1975

Ord. Chenopodietalia muralis Braun-Blanq. 1931

All. Chenopodion muralis Braun-Blanq. 1931

Ass. Comunidad de Chenopodium murale

Suball. Malvenion parviflorae Rivas Mart. 1978

Ass. Sisymbrio irionis-Malvetum parviflorae Rivas Mart. 1978

7. Clase ASPLENIETEA TRICHOMANIS (BRAUN-BLANQ. IN MEIER ET BRAUNBLANQ. 1934) OBERDORFER 1977

Ord. Asplenietea petrarchae Braun-Blanq. et Meier in Meier et Braun-Blanq. 1934

All. Teucrion buxifolii Rivas Goday 1955

Ass. Jasonio glutinosae-Teucrietum thymifolii Rigual, Esteve et Rivas-Goday 1972 corr.

Alcaraz et De la Torre 1988

Ord. Anomodonto-Polypodietalia serrulati O. Bolòs et Vives in O. Bolòs 1957

All. Polypodion serrulati Braun-Blanq. ex Braun-Blanq., Roussinine et Negre 1952

Ass. Polypodietum serrulati Braun-Blanq. ex Braun-Blanq., Roussinine et Negre 1952

8. Clase THLASPIETEA ROTUNDIFOLII BRAUN-BLANQ. 1947

Ord. Phagnaletalia saxatile Rivas Goday 1964

All. Melico-Phagnalion Rivas Goday et Esteve 1972

Ass. Euphorbio squamigerae-Dianthetum valentini De la Torre et Alcaraz in Alonso et al. 1997.

\section{Clase KOELERIO-CORYNEPHORETEA KIKLA IN KIKLA ET NOVAK 1941}

Ord. Sedo-Scleranthetalia Braun-Blanq. 1955

All. Sedion micrantho-sediformis Rivas Mart., Sánchez Gómez et Alcaraz in

Sánchez Gómez et Alcaraz 1993

Ass. Sedetum micrantho-sediformis O. Bolòs et Masalles in O. Bolòs 1981 subass. sedetosum glanduliferi De la Torre et al. inéd. 